\title{
LARGE EDDY SIMULATION OF SALTATION OVER GAUSSIAN HILLS
}

\author{
G. Huang , C. Le Ribault, S. Simoëns, I. Vinkovic, J.M. Vignon \\ LMFA, ECL, CNRS UMR 5509, UCB Lyon 1 \\ 36, avenue Guy de collongue, F 69134 Ecully Cedex, FRANCE \\ e-mail : catherine.le-ribault@ec-lyon.fr
}

Keywords: Large eddy simulation, Lagrangian models, saltation, hills

\begin{abstract}
Soil erosion plays an important role for climate, health and environmental issues. In this study, we focus on the transport of solid particles over a dry rough bed and their interaction with Gaussian hills. For this aim, we use large-eddy simulations (LES) performed with the atmospheric code ARPS. To follow the trajectories of the solid particles, a Lagrangian model is coupled with the LES, according to Vinkovic [1]. Different empirical models have been developed to take into account the interactions of particles with the rough bed, especially the takeoff, the rebound and the deposition.

A rough boundary layer at different external velocities is first simulated. The solid particle mass flux as function of the friction velocity is plotted and compared to classical analytical and empirical results in the Shields diagram.

Then, LES velocity and concentration profiles are compared to the experiments on solid particle dispersion downstream of Gaussian hills, by Simoëns et al. [2]. Special attention is given to the trapping of solid particles inside the recirculation bubbles. Regions of high particle deposition or erosion are identified. The influence of the distance between two consecutive hills is evaluated.
\end{abstract}




\section{INTRODUCTION}

Desertification and soil degradation are major issues for semi arid regions. Erosion is a very complex process which involves large spatial scales and their interaction with soils. Numerical simulations can play a very important role for detailed studies of erodible zones.

Obstacles and hills greatly modify flow characteristics of the incoming boundary layer and in some occasions create large recirculation zones where particles can be trapped and deposited. Bi-dimensional hills are widely used as idealized topographical elements in studies of flows over wall-mounted obstructions. Flows around various kinds of 2D hills have been studied in the past using analytical, numerical, laboratory and in-situ investigations ([3], [4], [5], [6]). Using wind-tunnel measurements, Cao and Tamura [4] highlighted the importance of the lee-side flow separation behind a steep hill.

Our aim is to evaluate the effects of recirculation zones generated by hills on the solid particle mass flux. To this purpose, an experimental campaign was conducted in the frame of the NFS/ANR Sino-French program PEDO-COTESOF "Particle EMission and Deposition Over Complex Terrain for Soil Fixation (PC09). Particle dispersion over one or two hills was investigated [2]. At our best knowledge, this is the only experimental case that produces both aerodynamic description, recirculation zones and solid particle concentration. Our purpose is to further study the link between the recirculation zone and particles deposition through numerical simulations.

The large eddy simulation approach allows the computation of instantaneous large turbulent structures able to produce ejections and sweeping events responsible for the takeoff and deposition of solid particles. This method, coupled with Lagrangian tracking of solid particles is particularly well suited to study saltation. LES coupled with a Lagrangian model has already been used for the study of sand saltation over a flat surface by [1] or [7].

Since the work of [8], research on saltation has concentrated on finding a description of the physical processes of interactions between the particles and the ground. The threshold velocity at which saltation is initiated was studied through windtunnel and in-situ experiments ([8], [9], [10], [11]). The behavior of rebounding and ejected particles was investigated through collision experiments between propelling solid particles and a static bed of similar particles ([12], [13]). Most of these models are presented in Shao [14]. Those studies have come to the parametrization of the ejection [15] or the rebound of particles from the surface [13]. From these models, a numerical method has been developed here to simulate solid particle transport and interaction with a rough bed with obstacles.

In the section 2, the flow governing equations and the numerical method are presented. Then, physical models related to particle motion are described. Computational results in the turbulent boundary layer and over one or several hills are discussed in the last section.

\section{LARGE EDDY SIMULATION AND NUMERICAL DETAILS}

Simulations are performed with The Advanced Regional Prediction System (ARPS version 5.15) code, developed at the university of Oklahoma for atmospheric flows 
[16]). The Navier-Stokes equations in the LES approach are filtered in order to separate the small scales from the large scales. The semi-compressible filtered Navier Stokes equations, including momentum, heat (potential temperature), mass (pressure) and the equation of state are solved. Subgrid scale turbulence is modeled through a 1.5 order turbulence closure scheme that requires the resolution of a subgrid scale kinetic energy equation (Yoshizawa \& Horiuti [17]).

A fourth-order finite difference method is used for the spatial integration of the equations. The time discretization is performed using a second-order implicit Crank-Nicholson method. The code is parallelized by a domain decomposition method. The full model equations and the numerical method are detailed in [16].

Since this study focuses on particle take-off and deposition, accurate wall modeling is very important for the quality of the simulations. A wall model, based on the equilibrium flow assumption have been initially developed in ARPS. Boundary conditions on the solid wall are imposed through surface fluxes of momentum. The roughness sublayer above rough surfaces presents complex interaction between wakes developing behind roughness elements. Based on direct numerical simulations and wind -tunnel experiments available in the literature, a generalized wall function for the roughness sublayer over periodical roughness elements has been developed by Huang et al. [18]. This law is used to estimate the friction velocity in the surface fluxes of momentum.

A method for generating three-dimensional, time-dependent turbulent inflow data for simulations of complex spatially developing boundary layers [19] is used to for the inlet conditions. The approach is based on extraction/rescaling techniques that produce instantaneous planes of velocity data from a downstream station far from the inlet.

\section{MOTION OF SOLID PARTICLES}

Equations for the motion of solid particles have been presented in [1]. Only the main features are summarized in this article.

\subsection{Lagrangian solid particles tracking}

The acceleration of the particle is determined by the forces acting on the particle. For the range of particles considered in this study, simplified equations including only the aerodynamic drag $F_{d}$ and the gravity forces $F_{g}$ are considered:

$$
\begin{gathered}
\frac{d \vec{x}_{p}}{d t}=\vec{v}_{p}(t) \\
\frac{d \vec{v}_{p}}{d t}=\frac{\vec{v}\left(\vec{x}_{p}(t), t\right)-\vec{v}_{p}(t)}{\tau_{p}} f\left(R e_{p}\right)+\vec{g}
\end{gathered}
$$

where $\vec{v}_{p}$ is the velocity of the particle, $\vec{v}\left(\vec{x}_{p}(t), t\right)$ is the velocity of the fluid at the particle position and $\vec{g}$ is the acceleration of gravity. The particle relaxation time is given by:

and

$$
\tau_{p}=\frac{\rho_{p} d_{p}^{2}}{18 \rho_{f} \nu}
$$

$$
R e_{p}=\frac{\left|\vec{v}_{p}-\vec{v}\right| d_{p}}{\nu}
$$


is the particle Reynolds number. Here, $d_{p}$ is the particle diameter, $\rho_{p}$ the particle density, $\rho_{f}$ the fluid density and $\nu$ the fluid viscosity. Effects of nonlinear drag are incorporated through $f\left(R e_{p}\right)$. In this work, an empirical relation is used [20].

$$
f\left(R e_{p}\right)= \begin{cases}1+0.15 R e_{p}^{0.687} & \text { if } \operatorname{Re}_{\mathrm{p}}<1000 \\ 0.0183 R e_{p} & \text { otherwise }\end{cases}
$$

The Lagrangian equations and the Navier Stokes equations are solved simultaneously and a second order Runge-Kutta scheme is used for the time integration. The different variables of the Navier-Stokes equations are only available at the discrete mesh nodes. They need to be interpolated on the trajectories of the fluid particles. A tri-linear scheme of quadratic Lagrange polynomials developed by Casulli \& Cheng [21], is used for the interpolation.

\subsection{Two-way coupling}

A two-way coupling model is used to take into account the influence of the solid particles on the fluid. Small particles, with much larger density than the surrounding fluid, act as if they were an extra burden to the fluid. The momentum transfer from particles to fluid is modeled by adding a drag force to the fluid momentum equation. This force is modeled as follows:

$$
\vec{f}=-\frac{1}{\rho_{f} V_{\text {grid }}} \sum_{p=1}^{N_{p}} m_{p} \frac{\vec{v}\left(\vec{x}_{p}(t), t\right)-\vec{v}_{p}(t)}{\tau_{p}} f\left(R e_{p}\right)
$$

where $V_{\text {grid }}$ is the volume of the fluid grid cell, $N_{p}$ is the number of numerically resolved particles in the grid cell and $m_{p}$ the mass of a particle.

\subsection{Take-off model}

To evaluate the aerodynamic entrainment rate, a new take-off criterion has been developed based on the instantaneous evaluation of the different forces exerted on the particle. The threshold friction velocity is the minimum friction velocity required for wind erosion to occur.

The driving force for the lift-off of sand particles is the aerodynamic lift $F_{l}$. According to Mollinger \& Nieuwstadt [22], the mean lift force can be expressed as:

$$
F_{l}=15.5 \rho \nu^{2}\left(\frac{u_{*}^{2} d_{p}}{\nu}\right)^{1.87}
$$

where $u_{*}$ is the friction velocity and $d_{p}$ is the diameter of the particle.

The gravity force $F_{g}$ and the inter-particle cohesive force $F_{a d}$ act as retarding forces. The gravity force is equal to:

$$
F_{g}=\frac{1}{6}\left(\rho_{p}-\rho_{f}\right) g d_{p}^{3}
$$

According to Zimon [23] the cohesive forces can be modeled as:

$$
F_{a d}=C d_{p}
$$

where $C$ is a parameter set to $C=1.43 \times 10^{-5} \mathrm{Nm}^{-1}$. 
The role of turbulent structures on the initiation of particle motion has to be accounted for as well. There is experimental evidence of the presence of sweep events at the time of grain entrainment. In our model, the particle lifts off when it is located within a sweep event and when the driving force is greater than the retarding forces.

$$
F_{l}>>+F_{a d}
$$

The friction velocity is estimated locally from the velocity fluctuations $u^{\prime}$ and $w^{\prime}$ by:

$$
u_{*}=\sqrt{u^{\prime} w^{\prime}}
$$

The experimental results of Diplat [10] showed that the duration of energetic near-bed turbulent events is also relevant in predicting grain entrainment. Thus, the product of the force by its duration, or impulse, is a more appropriate parameter for identifying flow conditions leading to particle take-off. Based on these conclusions, our criterion has been adapted to satisfy the following equation at each time step $t$ :

$$
\int_{t}^{t_{+} \tau_{p}}\left(F_{l}-F_{a d h}-P\right) d t>m_{p} v_{p 0}
$$

where the threshold lift-off velocity $v_{p 0}$ is equal to $v_{p 0}=\sqrt{2 g d_{p}}$.

\subsection{Rebound model}

Due to gravity, sand particles fall and impact the ground. Some of them remain on the ground, others rebound on the soil and can eject several new grains from the bed. In this study, the splash effects are not accounted for according to the experimental set-up.

The velocity after the rebound is characterized by its rebound angle $\theta_{r}$ and its norm $v_{r}$. They are modeled following a formulation proposed by Beladjine et al. [13]. The effect of local hill bed slope is also taken into account in the modeling of particle-wall collisions.

\section{EXPERIMENTAL SET-UP}

The flow field around one or two hills with various spacings was studied using particle image velocimetry (PIV) measurements. A Gaussian hill shape was used with a maximal slope on the lee side equal to $31^{\circ}$. With a model/field scale of 1 : 10000 , the experimental configuration is consistent with an atmospheric turbulent boundary layer of $150 \mathrm{~m}$ and an equivalent dune height of $20 \mathrm{~m}$. In the case of the experiments over two hills, two different spacings $3 H$ and $8 H, H$ being the hill height, were used. Three regimes were studied with external velocities ranging from $5.6 \mathrm{~m} / \mathrm{s}$ to $11.2 \mathrm{~m} / \mathrm{s}$. Experiments have been performed on both smooth and rough walls.

Powered by an upward-moving piston at a fixed place, particles were continuously introduced into the flow via a $20 \mathrm{~cm} \times 10 \mathrm{~cm}$ slot upstream of the Gaussian hills. The grains have a mean diameter of $200 \mu \mathrm{m}$ with variations between $170 \mu \mathrm{m}$ and $250 \mu \mathrm{m}$ and a density of $1000 \mathrm{~kg} / \mathrm{m}^{3}$. No splash effects were considered. Particle concentration and velocity profiles were obtained using digital image treatment. For more details on the experimental set-up, readers are referred to Simoëns al. [2]. 


\section{PARTICLE SALTATION IN A BOUNDARY LAYER}

To validate the particles transport models, simulations of turbulent boundary layers at different external velocities ranging from $5.6 \mathrm{~m} / \mathrm{s}$ to $20 \mathrm{~m} / \mathrm{s}$ are first performed. The mean diameter of the particles and their density are the same as in the experimental set-up.

Rough boundary conditions are applied at the wall. To mimic the experiment, the splash effect is not accounted for in our simulations and particles are lift through the take-off process. The saltation process is highly dependent on the friction velocity. The global behavior of particles in a turbulent flow can be characterized by the Shields number:

$$
S h=\frac{u_{*}^{2}}{\sigma_{p} g d_{p}}
$$

where $\sigma_{p}=\rho_{p} / \rho_{f}-1$. The Shields number measures the transport capacity of solid particles by the flow.

The saltation flux $Q$ is a crucial parameter for the study of wind erosion The streamwise mass flux is computed as follows:

$$
q_{x}(x, z)=\frac{1}{\Delta x_{p} L_{y} \Delta z_{p}} \Sigma_{p} m_{p} u_{p}
$$

where $m_{p}$ and $u_{p}$ are the mass and the streamwise velocity of the particles present in the volume $\Delta x_{p} L_{y} \Delta z_{p} . L_{y}$ is the length of the domain in the transversal direction. $\Delta x_{p}$ and $\Delta z_{p}$ are taken as $0.1 H$ where $H$ is the height of the boundary layer. The saltation mass flux, $\mathrm{Q}$, is the vertical integration of the streamwise flux $q_{x}$ :

$$
Q(x)=\int_{0}^{\infty} q_{x}(x, z) d z
$$

Various saltation models that relate the saltation flux to the characteristics of the flow and of the solid particles through empirical formulas are available in the literature (Bagnold [8], Cresseyls et al. [25], Owen [24], Sorensen [9]). Relationships of Bagnolds and Owen predict cubic dependence of $Q$ on the friction velocity whereas the models of Sorensen (1991) [9], and Creyssels et al. [25] predict a dependence on $u *^{2}$. On figure 1, simulation results are compared with the experiments and the different empirical formulas. The flux is normalized by the total flux $Q_{0}=\rho_{p} d_{p} \sqrt{g d_{p}}$. For low Shields numbers $(S h<0.1)$ a transitional zone appears starting from the threshold range of particle saltation. For high Shields numbers $(S h>0.1)$, our results exhibit a similar behavior to the empirical formula of Sorensen [9]. The absence of the splash effect in our simulation could explain the shift between our results and the other saltation models.

\section{PARTICLE SALTATION BEHIND GAUSSIAN HILLS}

Simulation results for the solid particles transport over one or two hills are now presented. Results are compared with the experimental results obtained with the external velocity of $U_{\infty}=11.2 \mathrm{~m} / \mathrm{s}$ on a rough wall. The computation domain includes an upstream Gaussian hill, $80 \mathrm{H}$ after the inlet and two Gaussian hills separated by a distance of $3 H$ or $8 H$. The center of the valley between the double hills 


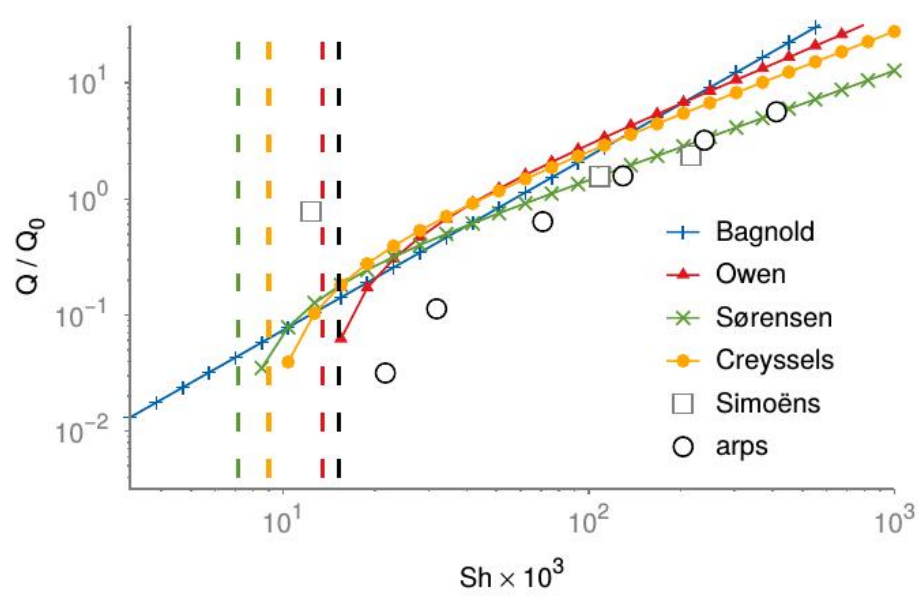

Figure 1: Saltation mass flux as a function of the Shields parameter

is located at $71 H$ after the first hill. Table 1 summarizes the domain size and the mesh resolution for all the simulations.

\begin{tabular}{|c|c|c|c|c|c|c|}
\hline$N_{x} \times N_{y} \times N_{z}$ & $L_{x} / H$ & $L_{y} / H$ & $L_{z} / H$ & $\Delta x / H$ & $\Delta y / H$ & $\Delta z_{\min } / H$ \\
\hline $1763 \times 63 \times 100$ & 176 & 6 & 15 & 0.1 & 0.1 & 0.05 \\
\hline
\end{tabular}

Table 1 Computational parameters

\subsection{Shear stress and recirculation zones}

The wall shear stress, which is of primary importance for the entrainment of solid particles, is presented in figure 2. A negative wall shear stress indicates the presence of a recirculation bubble. The friction velocity in regions where flow adheres to the wall is marked in red and backflow regions inside the recirculation zones are marked in blue. For the single hill configuration, a recirculation zone of approximatively $6 \mathrm{H}$ appears on the lee side of the hill. This is in agreement with the experimental results of Simoëns et al. [2]. The flow evolution before the first hill is similar in the three cases.

For the double hill configuration, two recirculation zones, respectively between and behind the two Gaussian hills appear. As the hill spacing increases from $3 \mathrm{H}$ to $8 \mathrm{H}$, we can see an expansion of the inter-hill vortex and a deflation of the leeside recirculation bubble. The size of the recirculation zone between the two hills is limited by the valley size. For the $8 H$ case, the second recirculation zone is smaller than for the $3 H$ case which is itself smaller than the recirculation zone behind the single hill. The double hill case with $3 H$ spacing behaves as much as a whole to the incoming flow and the effects of the double-hill configuration are negligible starting from $X / H=6$. 
(a)

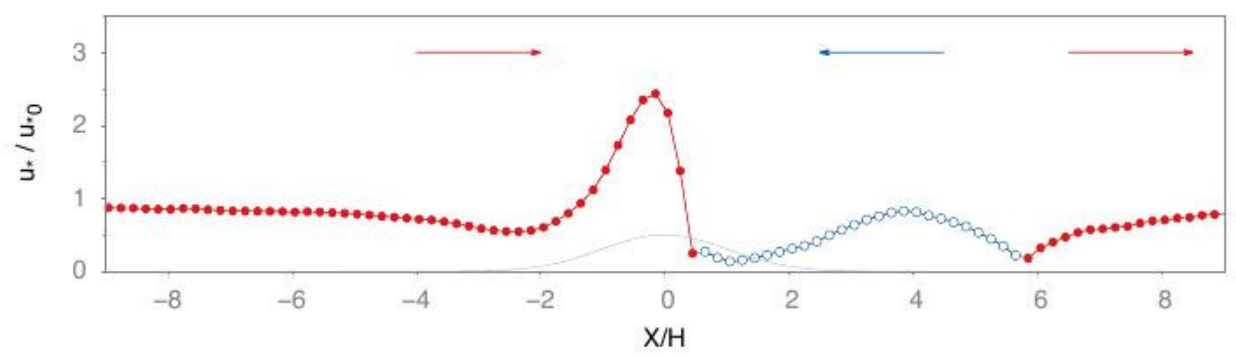

(b)

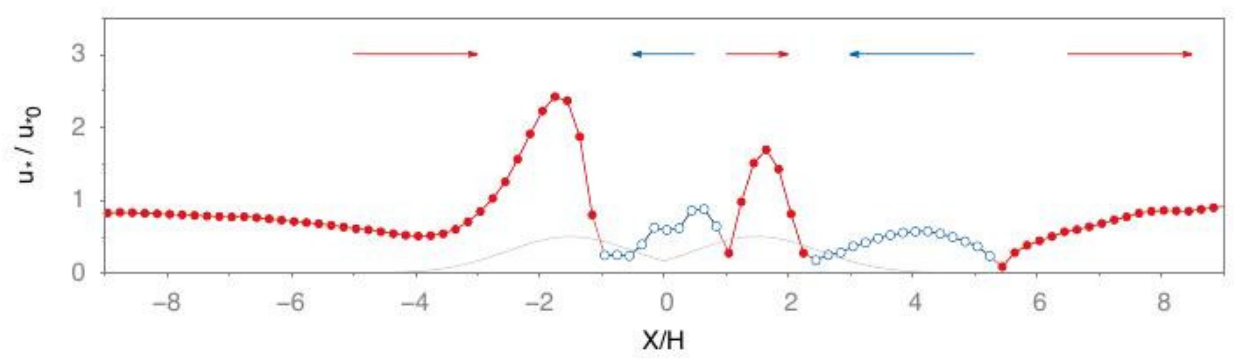

(c)

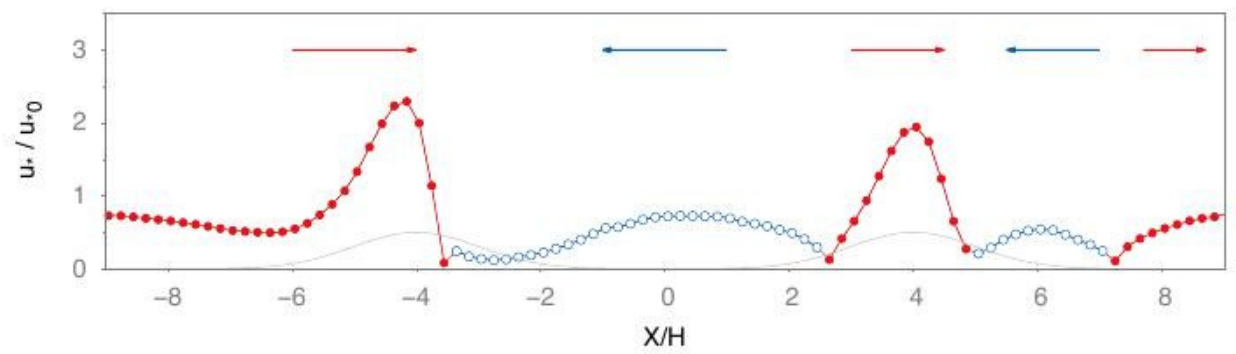

Figure 2: Shear velocity $u_{*}$ along the hill surface, (a) single hill, (b) $3 \mathrm{H}$, (c) $8 \mathrm{H}$

\subsection{Particles concentration}

Results for the particle concentration are presented for the single hill and for the two hills. For all the plots, the solid lines represent LES results whereas symbols represent experimental results. Profiles are normalized by the maximal concentration at each section. For the single hill, the coordinate origin is fixed at the center of the Gaussian hill and for the two hills, at the middle distance between the two hills. The concentration profiles describe how the particles react to the change of terrain from a flat surface to a steep, transverse hill.

The figure 3 presents the concentration profiles around one hill at different sections. The first profile at $x / H=-25$ corresponds to the concentration profile near the downstream edge of the sandbox. The other profiles present the concentration evolution around the hill. For all the profiles, the agreement between simulation and experimental results for the locations of the concentration peak is reasonable. The simulation seems to overestimate the saltation height growth behind the Gaussian hill. In the simulation results, a higher percentage of solid particles is moved into the upper part of the boundary layer behind the hill at $x / H=1.5$ and $x / H=3$. This upward migration is less pronounced in the experiments. Besides, in those zones, the simulation apparently underestimates the quantity of solid particles near 
the ground inside the recirculation zone compared to the experiments. This result could be explained by the fact that solid particles, impacting the windward side of the hill, are abruptly redirected upwards due to high hill slopes. As an inertial effect, these rebounding grains maintain this upward motion and reach high layers of rapid flow motion, which immediately evacuate them downstream of the hill.

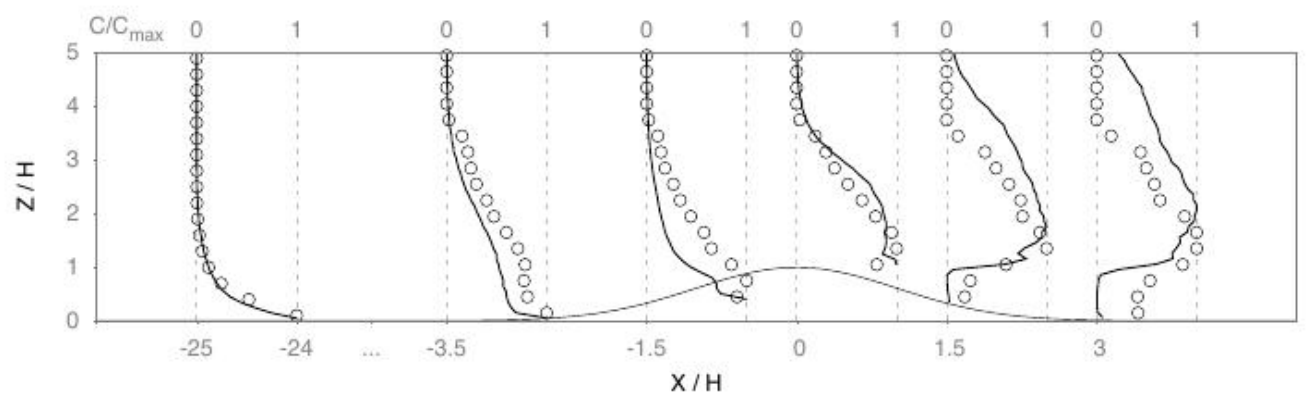

Figure 3: Concentration profiles at the end of the sandbox and around a single hill. Symbols experiments, lines - LES.

Figure 4 presents the same plots for the case of two consecutive hills separated by $3 H$. A better collapse with the experimental profiles is achieved around the second hill. Compared to experimental results, a smaller fraction of particles is present inside the wall vortex between the crests. The simulation overestimates the quantity of the incoming particles over the top of the first hill.

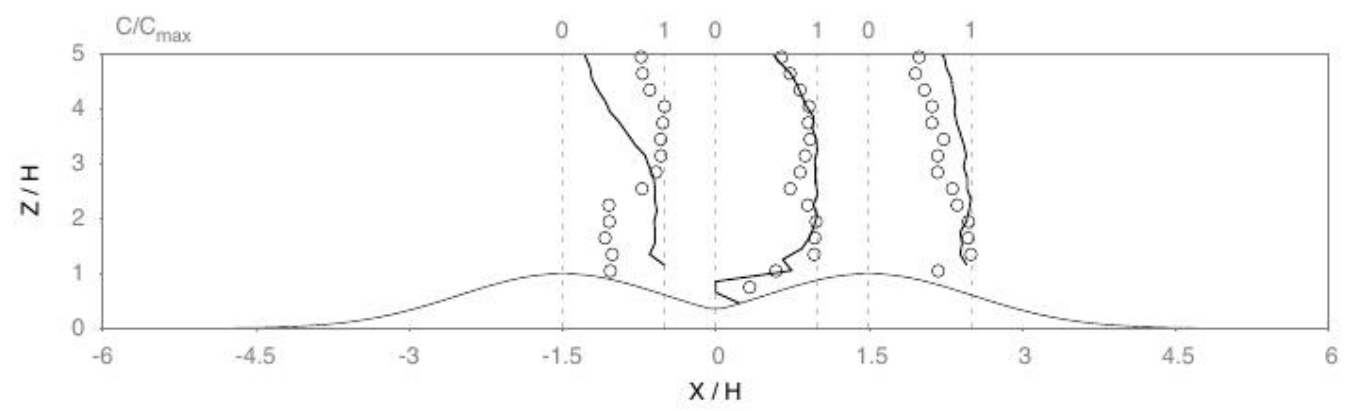

Figure 4: Concentration profiles for the $3 H$ case. Symbols - experiments, lines - LES.

In figure 5, the concentrations profiles around the two hills with $8 \mathrm{H}$ distance are plotted. Similar to figure 4, the simulation apparently underestimates the quantity of solid particles passing between the two hills compared to the experimental results. We note that the global evolution of concentration peaks is similar between the $3 H$ and the $8 H$ cases. This implies a weak influence of the hill spacing on the overall evolution of mean particle concentration.

\subsection{Near wall particle transport and deposition}

Our goal is to explore the link between particle trapping and the flow characteristics inside the recirculation zones. To this purpose, regions of preferential deposition 


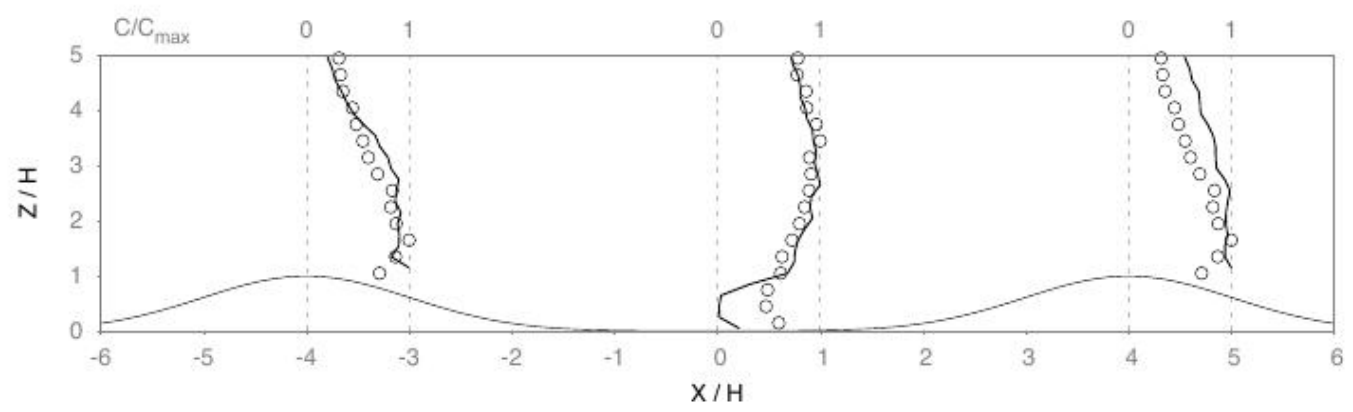

Figure 5: Concentration profiles for the $8 H$ case. Symbols - experiments, lines - LES.

in the recirculation zones between and after the hills are identified. The net deposition of particles is defined as the accumulation $\lambda_{D}$ scaled by the packing density at the sandbox $\lambda_{b o x}$. The net deposition is computed during a period of $\Delta T=30 \delta_{0} / u_{*}$, $\delta_{0}$ being the depth of the incoming boundary layer. Regions with $\lambda_{D}<0$ indicate net erosion, whereas $\lambda_{D}>0$ indicate net deposition. An instantaneous map of the net accumulation is shown in Figure 6 for the three different hill spacings. Areas of erosion are marked in red and deposition zones are marked in yellow.

The sporadic areas of net erosion are weak and more likely to occur on the windward face of the hill. The net deposition of particles is more important and most of the time located in the recirculation zones behind and between hill crests. The accumulations due to the deposited grains are in general one, even two orders of magnitude sparser than that at the sandbox. Not surprisingly, the regions of high deposition are located at the junction of positive and reverse transport, where the friction velocity and the mean lift force are weak. This could be explained by the fact that instantaneously frequent alterations take place in the direction of particle transport in these areas. For the particles already trapped by the recirculation zones, this back and forth motion may lead to a final deposition. Due to a weak lift force, particles spend a longer time immobilized on the wall and therefore contribute to the net accumulation in these regions.

\section{CONCLUSIONS}

The paper presents a numerical study of the physical problem of soil erosion by wind. The particle transport inside a turbulent boundary layer in the presence of obstacles is studied to improve our knowledge on wind erosion and particle deposition over complex terrains.

The simulation results are compared to the PC09 experiments of Simoëns et al. [2]. The flow between and behind Gaussian hills is dominated by large recirculation zones. The predicted particle concentration is in overall agreement with the experiments. A mapping of particle accumulation on the wall reveals preferential particle deposition at the junction between regions with mean and adverse particle transport.

\section{Acknowledgments}

We acknowledge NFSC/ANR Chinese/French program PEDO-COTESOF. This work was granted access to the HPC resources of CINES. Numerical simulations 

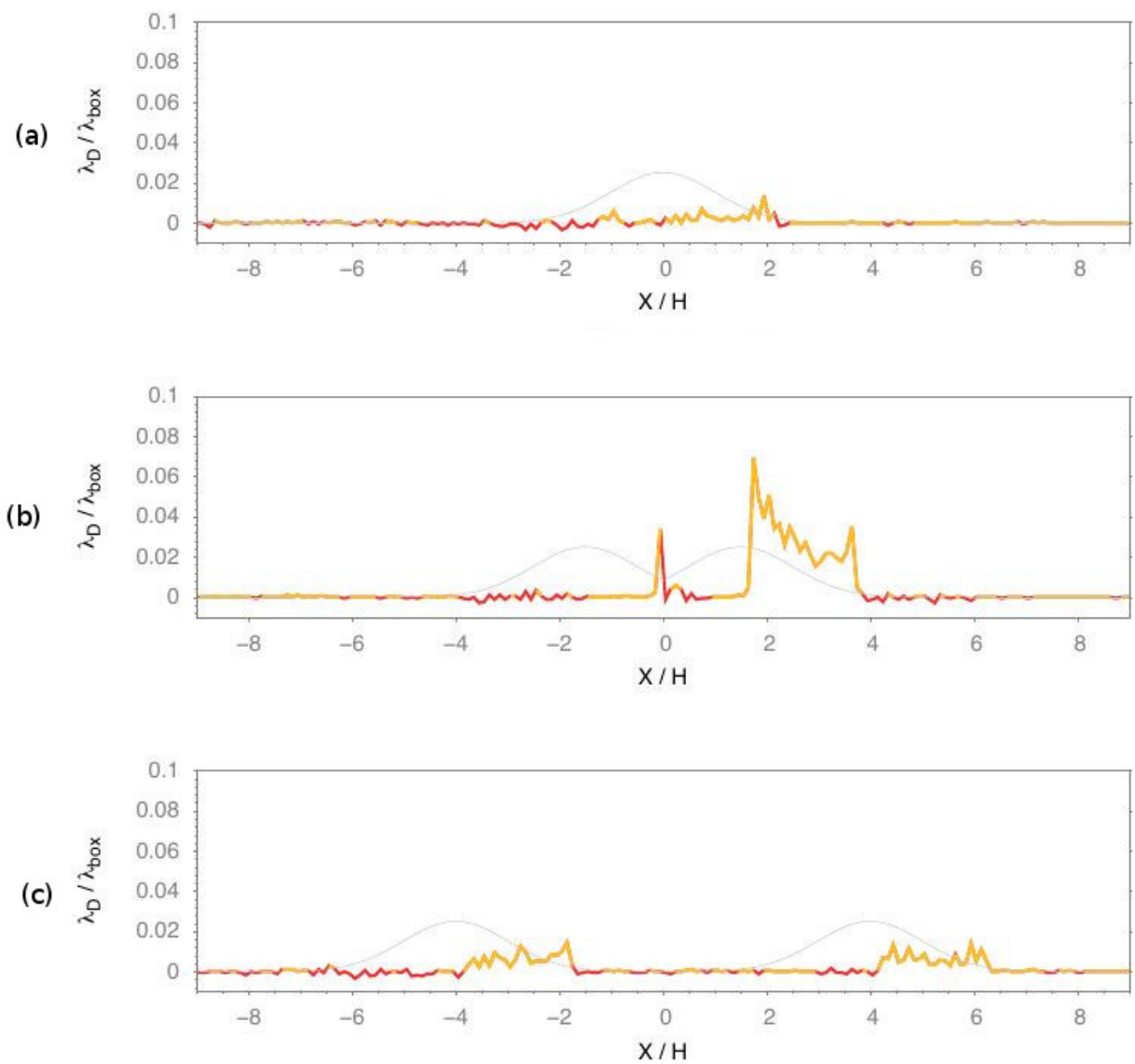

Figure 6: Instantaneous map of the net accumulation of solid particles. Red - erosion, yellow deposition., (a) single hill, (b) $3 \mathrm{H}$, (c) $8 \mathrm{H}$

were also performed on the P2CHPD parallel cluster.

\section{REFERENCES}

[1] Vinkovic I., Aguirre C., Simoëns S., Ayrault M. Large eddy simulation of the dispersion of solid particles in a turbulent boundary layer. Boundary Layer Meteoroly 121, 283-311, 2006

[2] Simoëns S. , Saleh S., Le Ribault C. , Belmadi M., Zegadi R., Allag F., Vignon J.M., Huang G., "Influence of Gaussian hill on concentration of solid particles in suspension inside Turbulent Boundary Layer", Procedia IUTAM, 17 , pp. $110-118,2015$

[3] Jackson P.S., Hunt J.C.R. Turbulent wind flow over a low hill, Quarterly Journal of the Royal Meteorological Society, 101, 929-955, 1975

[4] Cao S., Tamura T. Effects of roughness blocks on atmospheric boundary layer flow over a two-dimensional low hill with/without sudden roughness change, Journal of wind engineering and industrial aerodynamics, 95, 679-695, 2007 
[5] Chapman, C., Walker, I.J., Hesp, P.A., Bauer, B.O., Davidson-Arnott, R.G.D. Oller- head, J. (2013). Reynolds stress and sand transport over a foredune. Earth Surface Processes and Landforms, 38, 17351747, 2013

[6] Almeida G.P., Durao D.F.G., Heitor M.V. Wake flows behind two-dimensional model hills, Experimental Thermal and fluid Science, 7, 87-101, 1993

[7] Dupont S., Bergametti G., Marticorena B., Simoëns S. Modeling saltation intermittency, Journal of Geophysical Research: Atmospheres, 118, 7109-7128, 2013

[8] Bagnold R.A. The physics of wind blown sand and desert dunes, Methuen, London, 1941

[9] Sorensen M. An analytic model of wind-blown sand transport Acta Mechanica Supplementum, 67-81, Springer Vienna, Vienna, 1991

[10] Diplat P., Dancey C.L. Coherent flow structures, initiation of motion, sediment transport and morphological feedbacks in rivers. J.G. Venditti, J.L. Best, M. Church \& R.J. Hardy eds., Coherent Structures at Earth's surface, 289-307, John Wiley \& Sons, Ltd, Chichester,UK, 2013

[11] Foucaut J.M., Stanislas M. Take-off threshold velocity of solid particles lying under a turbulent boundary layer, Experiments in Fluids, 20, 1996

[12] Anderson R.S., Haff P.K, Wind modification and bed response during saltation of sand in air, Aeolian Grain Transport 1, Springer Vienna, Vienna, pp. 21-51, 1991

[13] Beladjine D., Ammi M., Oger L., Valance A. Collision process between an incident bed and a three-dimensional granular packing, Physical Review E., 75, 061305, 2007

[14] Shao Y. Physics and modeling of wind erosion, 37, Atmospheric and Oceanographic Sciences Library, Springer Netherlands, Dordrecht, 2009

[15] Descamps I., Erosion éolienne d'un lit de particules à large spectre granulométrique, Thèse de doctorat, Université de Valenciennces et du HainautCambrésis, France, 2004

[16] Xue M., Droegemeier K., Wong V., Shapiro A., Brewster K. ARPS version 4.0 Users Guide, 1995

[17] Yoshizawa A., Horiuti K. A statistically-derived subgrid-scale kinetic models for large eddy simulation of turbulent, J. Phys. Soc. Japan 54(8), 2834-2839, 1985

[18] Huang G., Simoëns S., Vinkovic I., Le Ribault C., Dupont S., Bergametti G. Law-of-the-wall in a boundary-layer over regularly distributed roughness elements, Journal of Turbulence, 2016 
[19] Lund T., Wu X., squires K Generation of turbulent inflow data for spatially developing boundary layer simulations, Journal of Computational Physics, 140, 233-258, 1998

[20] Clift R., Grace J.R., Weber M.E., Bubbles, drops and particles, Academic Press, New York 1978

[21] Casulli V., Cheng R.T. Semi-implicit finite difference methods for the threedimensional shallow water flow, International Journal for Numerical in Fluids, 15, pp. 629-648, 1992

[22] Mollinger A.M., Nieuwstadt F.T.M. Measurement of the lift force on a particle fixed to the wall in the viscous sublayer of a fully developed turbulent boundary layer,Journal of Fluid Mechanics, 316, 285-306, 1996

[23] Zimon A.D. Adhesion of dust and powder, Springer, 1969

[24] Owen P.R. Saltation of uniform grains in air, Journal of fluid Mechanics, 20, 225-242, 1964

[25] Creyssels M., Dupont P., Ould El Moctar, Valance A., cantat A., Jenkins J.T., Pasini J.M., Rasmussen K.R., Saltating particles in a turbulent boundary layer: experiment and theory, Journal of Fluid Mechanics, 625, 47-28, 2009 